\title{
ERRATUM
}

\section{Emerging regulation and functions of autophagy}

\section{Patricia Boya, Fulvio Reggiori and Patrice Codogno}

Nat. Cell Biol. 15, 713-720 (2013); corrected after print 1 July 2013

In the print version of this Review, Table 2 (shown below) was mistakenly omitted. It appears correctly in the HTML and PDF versions.

\begin{tabular}{|c|c|c|}
\hline Gene & Autophagy-independent pathways & References \\
\hline ULK1 & Brucella vacuole biogenesis & 80 \\
\hline ATG3 & Mitochondrial homeostasis (conjugated to Atg12) & 38 \\
\hline ATG4B & Osteoclast bone resorption & 54 \\
\hline ATG5 & $\begin{array}{l}\text { Osteoclast bone resorption; phagocytosis; IFN- } \alpha / \text { IFN- } \beta / \text { IFN- } \gamma \text { antiviral response (conjugated to Atg } 12 \text { ); } \\
\text { pro-apoptotic role }\end{array}$ & $54,55,77,78,84,94$ \\
\hline Beclin 1 & Phagocytosis; Brucella vacuole biogenesis & 80,95 \\
\hline ATG7 & Osteoclast bone resorption; phagocytosis; IFN- $\gamma$ antiviral response & $54,55,78,94$ \\
\hline LC3 & Osteoclast bone resorption; tuning of endoplasmic-reticulum-associated degradation; coronavirus infection & $54,55,81,82$ \\
\hline ATG12 & $\begin{array}{l}\text { Mitochondrial apoptosis; IFN- } \alpha / \text { IFN- } \beta / \text { IFN- } \gamma \text { antiviral response (conjugated to Atg5); } \\
\text { mitochondrial homeostasis (conjugated to Atg3) }\end{array}$ & $38,77,78,83$ \\
\hline ATG14 & Brucella vacuole biogenesis & 80 \\
\hline ATG16 & IFN- $\gamma$ antiviral response & 78 \\
\hline p150 & Brucella vacuole biogenesis & 80 \\
\hline Ptdlns3PKC3 & Phagocytosis; Brucella vacuole biogenesis & 80,94 \\
\hline
\end{tabular}

